\title{
Hyperbaric oxygen therapy for postoperative ischemic bronchitis after resection of lung cancer
}

\author{
Makoto Endoh ${ }^{1,2}$, Hiroyuki Oizumi ${ }^{2}$, Hirohisa Kato ${ }^{2}$, Jun Suzuki ${ }^{2}$, Hikaru Watarai ${ }^{2}$, Akira Hamada ${ }^{2}$, \\ Katsuyuki Suzuki ${ }^{1}$, Kenta Nakahashi ${ }^{2}$, Satoshi Shiono ${ }^{1}$, Mitsuaki Sadahiro ${ }^{2}$ \\ ${ }^{1}$ General Thoracic Surgery, Yamagata Prefectural Central Hospital, Aoyagi, Yamagata City, Yamagata Prefecture, Japan; ${ }^{2}$ Department of Surgery II, \\ Faculty of Medicine, Yamagata University, Iida-Nishi, Yamagata City, Yamagata Prefecture, Japan \\ Contributions: (I) Conception and design: M Endoh, H Oizumi; (II) Administrative support: S Shiono, M Sadahiro; (III) Provision of study materials \\ or patients: M Endoh, H Kato, J Suzuki, H Watarai, A Hamada, K Suzuki, K Nakahashi; (IV) Collection and assembly of data: M Endoh; (V) Data \\ analysis and interpretation: H Oizumi, M Endoh; (VI) Manuscript writing: All authors; (VII) Final approval of manuscript: All authors. \\ Correspondence to: Hiroyuki Oizumi, MD, PhD. Department of Surgery II, Faculty of Medicine, Yamagata University, 2-2-2 Iida-Nishi, Yamagata \\ City 990-9585, Yamagata Prefecture, Japan. Email: hohizumi@med.id.yamagata-u.ac.jp.
}

\begin{abstract}
Background: Hyperbaric oxygen therapy (HBOT) has been used successfully in the treatment of specific ischemic injuries, but has been a little evaluated specifically in postoperative ischemic bronchitis (POIB). The purpose of this study was to evaluate the effect of HBOT when used for POIB after resection of lung cancer. Methods: From January 1999 to December 2016, 1,100 patients underwent lymph node dissection (LND) and either anatomic pulmonary resection or lung resection with bronchoplasty for lung cancer. POIB was diagnosed by bronchoscopy. HBOT was administered after POIB was diagnosed. HBOT comprised one 60-minute session daily in the hyperbaric chamber at 2.0 absolute atmospheres with $100 \%$ oxygen. We retrospectively analyzed the clinical course, timing of onset of POIB, outcomes, and any adverse events.

Results: Seven patients were identified to have had POIB treated with HBOT, all of whom were men with a smoking history and a median age of 65 years (range, 57-72 years). The operative procedures included three lung resections with bronchoplasty, three right lower lobectomies, and one right middle lobectomy performed owing to torsion of the middle lobe after right upper sleeve lobectomy. All 7 patients underwent subcarinal LND. POIB was diagnosed at a median of 11 days (range, 4-41 days) postoperatively. The median duration of an HBOT session was 7 days (range, 3-11 days). POIB resolved in 5 patients but worsened in 2, both of whom required further surgery.
\end{abstract}

Conclusions: Prospective clinical trials are now needed to confirm the potential benefits of HBOT in POIB.

Keywords: Bronchial fistula; hyperbaric oxygen therapy (HBOT); ischemic bronchitis; lung cancer

Submitted Jul 10, 2018. Accepted for publication Oct 12, 2018.

doi: $10.21037 /$ jtd.2018.10.45

View this article at: http://dx.doi.org/10.21037/jtd.2018.10.45

\section{Introduction}

The importance of peribronchial tissue in wound healing is well established, and the extent of lymph node dissection (LND) in lung cancer surgery may compromise bronchial microvascularization and negatively influence the process of bronchial stump healing $(1,2)$. Ischemic change in bronchial stumps after lung cancer surgery is considered as one of several possible etiologies contributing to the development of bronchopleural fistula (BPF), which remains a major complication (3-6). While postoperative ischemic bronchitis (POIB) has been well characterized and classified after lung transplantation (1), few reports on this complication after pulmonary resection for lung cancer have been published. Impaired healing of bronchial stumps is a constant impediment to successful surgical procedures performed for lung cancer and is thought to be caused by ischemia (7). Successful healing of bronchial stumps and anastomoses 
is of critical importance after major lung resection and reconstruction of the airway tract.

Hyperbaric oxygen therapy (HBOT) has been used successfully in the treatment of specific ischemic injuries, but has not been fully evaluated in POIB. HBOT accelerates wound healing by promoting epithelization and neovascularization $(8,9)$. Experimental studies have demonstrated the positive effects of HBOT on healing of the anastomotic suture line after primary tracheal anastomosis $(10,11)$. Recent clinical application based on these findings indicate that HBOT promotes complete healing in patients with complications affecting the bronchial stump and anastomosis (12-14).

On the basis of experimental studies on ischemia and healing of the airway tract, we hypothesized that HBOT may be effective in POIB. The purpose of this study was to evaluate our clinical experience of the effect of HBOT when used specifically for POIB after resection of lung cancer.

\section{Methods}

\section{Patients}

From January 1999 to December 2016, 1,100 patients underwent LND and either anatomic pulmonary resection or lung resection with bronchoplasty for lung cancer at Yamagata University Hospital. Routine systematic nodal dissection of the hilar and mediastinal lymph nodes was performed in all cases. POIB was diagnosed by fiberoptic bronchoscopy as indicated in the presence of signs suggestive of BPF (fever, hyperleukocytosis, and altered sputum). We defined POIB as true ischemia, that is, loss of mucosal sheen and either yellowish or fibrin deposit, and necrosis, that is, a mucosal defect and/or ulceration. The clinical and surgical parameters assessed were smoking history, comorbidity, operative procedure, histologic diagnosis of the resected specimens, and pathologic staging. The smoking history was described as the pack-years smoked (the average number of packages of cigarettes smoked per day multiplied by the number of years when the individual smoked). Staging definitions for TNM components were according to the 8th edition of the International Staging System for Lung Cancers (15). The study was approved by the institutional review board, and each patient gave written informed consent before bronchoscopic examination.

\section{Treatment by $\mathrm{HBO}$}

HBOT was administered after diagnosis of POIB if there were no contraindications (16). HBOT consisted of one 60-minute session per day in the hyperbaric chamber at 2.0 absolute atmospheres with $100 \%$ oxygen. The duration of the prescribed therapy was usually 7 days. Iterative fiberoptic bronchoscopy was performed to monitor evolution of the ischemic injury. We usually check status of the bronchial mucosa using bronchoscopy at 1 week after surgery. If ischemic change was found there, we commonly perform repeating bronchoscopy every week until recovery. Patients received HBOT were evaluated by scope at 1 , 3, 6 and 12 months after recovery. Biopsy from ischemic mucosa was not performed during bronchoscopy. We retrospectively analyzed the clinical course of each patient, the timing of onset of POIB, and the outcomes as well as any adverse events of HBOT.

\section{Statistical analysis}

The statistical analysis was performed using the JMP 13.0 software package (SAS Inc., Cary, NC, USA). Among quantitative variables, categorical ones are expressed as $\mathrm{n}(\%)$ and continuous variables as the mean \pm standard deviation or as the median and range.

\section{Results}

We identified 7 patients with POIB that had been treated with HBOT, all of whom were men with a history of smoking. Their median age was 65 years (range, $57-72$ years) (Table 1). Five patients $(71 \%)$ had several risk factors for BPF. All tumors were over $3 \mathrm{~cm}$ in size. Two were pathologic stage IB, 4 were stage IIB, and 1 was stage IIIA. The histologic types were as follows: 5 squamous cell carcinomas (71\%), 1 adenocarcinoma (14\%), and 1 small cell carcinoma (14\%). Complete resection was performed in all patients.

All surgical procedures were right-sided, and included three lung resections with bronchoplasty (43\%), three lower lobectomies (43\%), and one middle lobectomy that was performed owing to a torsion of the middle lobe after upper sleeve lobectomy (14\%; Table 2). All 7 patients underwent subcarinal LND. POIB was found at a median of 11 days (range, 4-41 days) postoperatively. All these patients had fever and hyperleukocytosis $\left(>9,000 / \mathrm{mm}^{3}\right)$ and elevation of 
Table 1 Patient demographics and clinical characteristics

\begin{tabular}{lllllllll}
\hline Patients No. & Onset & Sex & Age & Pack-years & Comorbidity & Histology & cTNM & pTNM \\
\hline 1 & 1999 & Male & 61 & 83 & LrC, HT & SQ & $2 \mathrm{a} 00$ & $2 \mathrm{a} 10$ \\
2 & 2000 & Male & 57 & 70 & - & SQ & $1 \mathrm{~b} 00$ & $2 \mathrm{a} 00$ \\
3 & 2003 & Male & 65 & 45 & - & SQ & $2 \mathrm{a} 20$ & $2 \mathrm{a} 10$ \\
4 & 2004 & Male & 72 & 27 & LC, HT & SCLC & 310 & $2 \mathrm{a} 10$ \\
5 & 2007 & Male & 72 & 80 & DM, AP, HT & SQ & $2 \mathrm{a} 10$ & $2 \mathrm{a} 00$ \\
6 & 2015 & Male & 68 & 20 & AT, ileus, HT & SQ & 300 & $2 \mathrm{a} 10$ \\
7 & 2016 & Male & 61 & 82 & DM & AD & $2 \mathrm{a} 20$ & $1 \mathrm{a} 20$ \\
\hline
\end{tabular}

$\mathrm{AP}$, angina pectoris; AT, adrenal tumor; DM, diabetes mellitus; $\mathrm{HT}$, hypertension; LC, lung cancer; LrC, laryngeal cancer; SQ, squamous cell carcinoma; SCLC, small cell lung cancer.

Table 2 Operative procedure, hyperbaric oxygen therapy, and outcome

\begin{tabular}{|c|c|c|c|c|c|c|c|}
\hline $\begin{array}{l}\text { Patients } \\
\text { No. }\end{array}$ & $\begin{array}{l}\text { Operative } \\
\text { procedure }\end{array}$ & Coverage & $\begin{array}{l}\text { Localization } \\
\text { of IB }\end{array}$ & $\begin{array}{l}\text { Ischemia or } \\
\text { necrosis }\end{array}$ & $\begin{array}{l}\text { Onset of } \\
\text { IB (POD) }\end{array}$ & $\begin{array}{l}\text { Duration of } \\
\text { HBOT (days) }\end{array}$ & Outcome \\
\hline 1 & RUsL & $\mathrm{ICM}$ & Anastomosis & Ischemia & 4 & 8 & Improved \\
\hline 3 & RML after RUsL & $\mathrm{ICM}$ & Stump & Necrosis & 41 & 7 & Improved \\
\hline 5 & RLL & $\mathrm{ICM}$ & Stump & Necrosis & 7 & 4 & $\begin{array}{l}\text { Terminated for tympanic } \\
\text { barotrauma; requiring surgical } \\
\text { procedure }\end{array}$ \\
\hline 6 & $R U L+S 6 s R$ & $\mathrm{ICM}$ & Anastomosis & Necrosis & 40 & 10 & Improved \\
\hline
\end{tabular}

HBOT, hyperbaric oxygen therapy; IB, ischemic bronchitis; ICM, intercostal muscle flap; POD, postoperative day; PP, parietal pleural flap; RLL, right lower lobectomy; RMB, right main bronchus; RML, right middle lobectomy; RS6sS, right S6 (superior segment) sleeve segmentectomy; RUsL, right upper sleeve lobectomy; RUL + S6sR, right upper lobe and S6 (superior segment) sleeve resection.

serum C-reactive protein.

The POIB was localized to the bronchial stump in 4 patients $(57 \%)$ and to the bronchial anastomosis in 3 patients (43\%). POIB was observed at multiple sites, including the bronchial stump, intermediate bronchus, and right main bronchus in one patient (14\%), but without extension to the contralateral bronchial tree (Figure 1). On bronchoscopy, ischemia was observed in all patients, with partial necrosis identified in cases 2-7. In case 4, bronchial ischemia and partial necrosis of the anastomosis after bronchoplasty following right S6 segmentectomy and resection of the intermediate bronchus on postoperative day (POD) 6 improved after 7 sessions of HBOT (Figure $1 A, B, C$ ). In case 5 , partial necrosis localized at the bronchial stump after right lower lobectomy was found on POD 6, and the bronchial ischemia partially improved after only 4 sessions of HBOT; however, the lesion evolved into necrosis and BPF that needed surgical intervention to terminate because of tympanic barotrauma (Figure 1D,E,F). Cases 6 and 7 indicated bronchial mucosal ischemia, which was not directly dealt in segmental bronchi or the right main bronchus (Figure 2).

In case 6 , the most severe ischemic area was observed not at the anastomosis but at the bronchial wall on the distal side at the orifice of the right $\mathrm{B} 8, \mathrm{~B} 9$, and $\mathrm{B} 10$ on POD 158 (Figure $2 A$ ). The bronchial wall had partially disappeared in the peripheral areas of B8, B9 and B10, so pulmonary parenchyma was seen through the bronchus, with an 

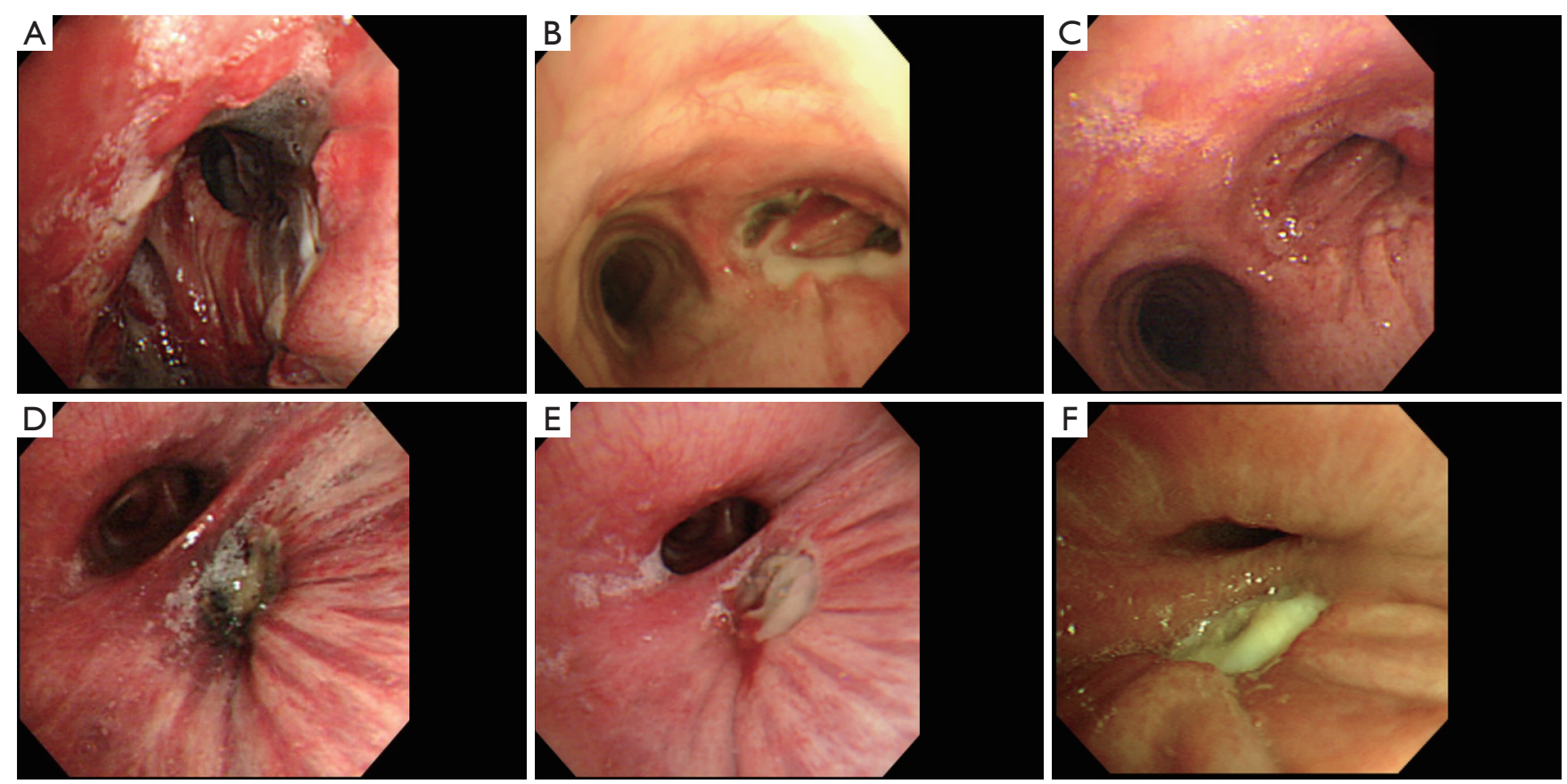

Figure 1 Changes in bronchial ischemia after hyperbaric oxygen therapy. (A) Bronchial ischemia and partial necrosis of the anastomosis on postoperative day 6 in case 4; (B) the area of postoperative ischemic bronchitis shrank after 7 sessions of hyperbaric oxygen therapy on postoperative day 13; (C) the bronchial mucosa had improved by postoperative day 52; (D) partial necrosis at the bronchial stump on postoperative day 6 in case 5; (E) the area of postoperative ischemic bronchitis was localized and the bronchial ischemia was partially improved after only 4 sessions of hyperbaric oxygen therapy on postoperative day 13 . Hyperbaric oxygen therapy was terminated in this patient because of tympanic barotrauma; (F) the lesion evolved into necrosis and bronchopleural fistula that needed surgical intervention.

internal fistula evident between the impaired bronchus and pleural cavity (Figure 2B,C). In case 7, ischemia and partial necrosis were observed at the right main bronchus after right lower lobectomy on POD 6 (Figure 2D). The bronchial mucosa had improved to localized ischemia after 11 sessions of HBOT on POD 27 (Figure 2E).

The median duration of the HBOT sessions was $7 \pm 3$ days (range, 3-11 days). The POIB resolved after HBOT in 5 patients $(71 \%)$. Two patients experienced worsening POIB after HBOT and required a further surgical procedure. One patient developed tympanic barotrauma without any functional consequences after 4 days and could not continue HBOT. However, 9 days after this patient was discharged, his POIB was found to have worsened and bronchoscopic findings were suspicious for $\mathrm{BPF}$, so a right middle lobectomy with resection of the bronchus intermedius was performed. Another patient developed BPF 3 days after starting HBOT and required thoracostomy for subsequent empyema.

The median postoperative hospital stay was 28 days (range, 15-135 days) and the median hospital stay after
HBOT was 12 days (range, 7-98 days). There was no postoperative mortality.

\section{Discussion}

In this study, our hypothesis was that HBOT might be a useful adjunct therapy for healing of the bronchial stump in patients who develop POIB following lung cancer surgery by preventing worsening of necrosis of bronchial tissue and $\mathrm{BPF}$, thereby avoiding the need for surgical intervention. Thus, HBOT could be considered as a therapeutic option if ischemic bronchitis is observed at the bronchial anastomosis or stump during postoperative bronchoscopic surveillance.

Ischemic bronchitis is a frequent complication after lung cancer surgery and can lead to potentially lifethreatening complications, such as BPF or anastomotic failure $(3,4)$. Satoh et al. found that the incidence of POIB after lung cancer resection was $2.5 \%(29 / 1,015)(17)$. According to that report, the most common site was the right intermediate trunk stump $(7.1 \%)$, followed by the left upper (3.4\%), right lower (3.4\%), right middle (3.3\%), 

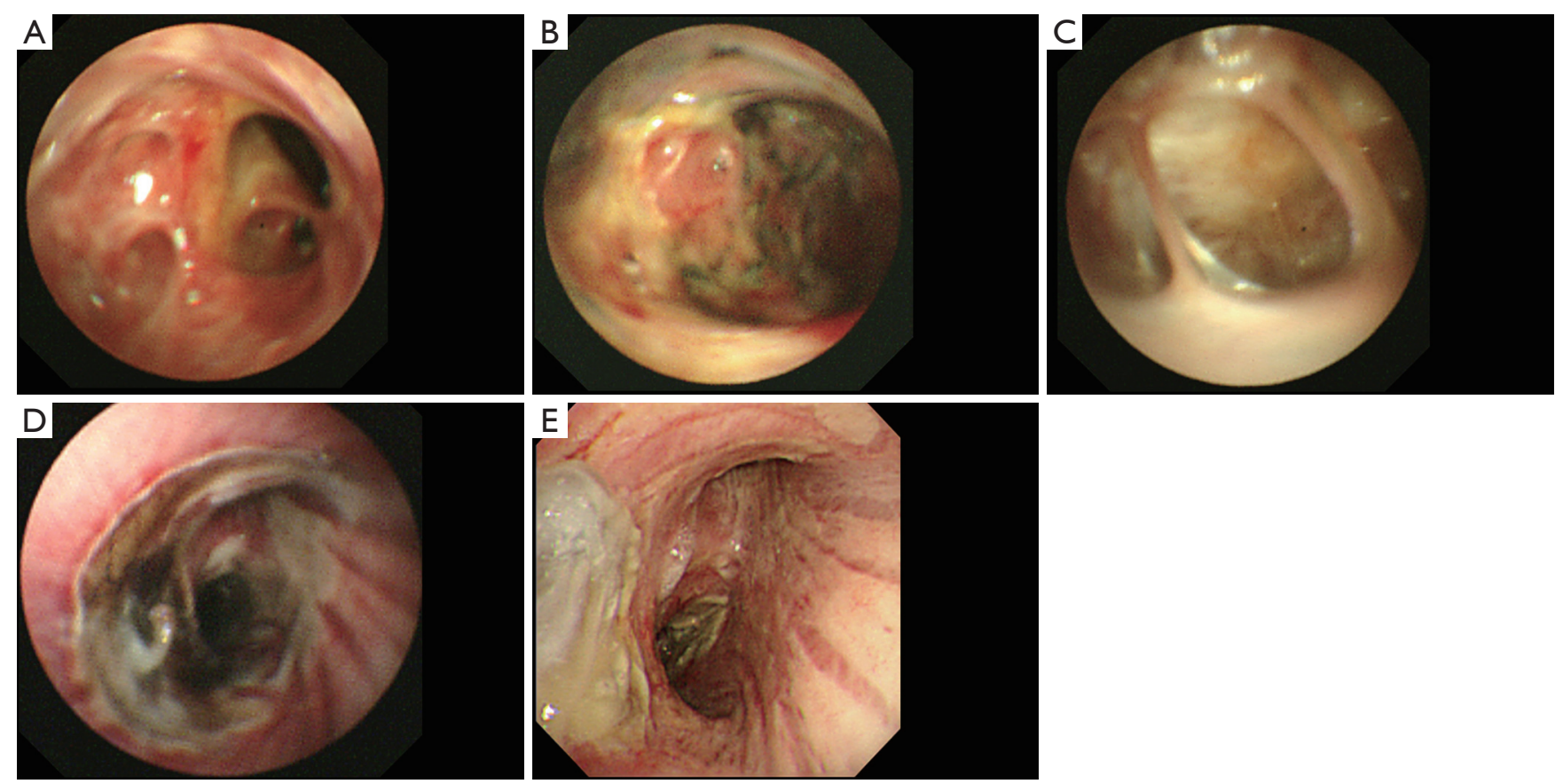

Figure 2 Bronchial alterations after surgery. (A) The most severe ischemic area was observed not at the anastomosis but on the bronchial wall of the distal side at the orifice of right B9 and B10 on postoperative day 158 in case 6; $(\mathrm{B}, \mathrm{C})$ the bronchial wall in the peripheral area of B9 and B10 had partially disappeared, so the pulmonary parenchyma could be seen through the bronchus and an internal fistula of communication was formed between impaired bronchus and pleural cavity; (D) bronchial ischemia and partial necrosis at the right main bronchus after right lower lobectomy on postoperative day 6 in case 7; (E) the bronchial mucosa had improved to localized ischemia after 11 sessions of hyperbaric oxygen therapy on postoperative day 27.

and left lower (3.2\%) lobar bronchial stump. BPF occurred in $3(10 \%)$ of 29 patients with POIB and in $4(0.4 \%)$ of 986 patients without POIB. Being male, a smoker, having diabetes mellitus, having postoperative respiratory complications, and undergoing subcarinal LND were found to have a significant impact on the risk of POIB. Benhamed et al. reported that POIB developed in 34 (3.2\%) of the 1,071 patients in their series (12). They also reported that POIB was asymptomatic in $80 \%$ of cases and was accompanied by fever and hyperleukocytosis in $20 \%$. In our series, all 7 patients had symptoms and were diagnosed to have POIB on bronchoscopy; 6 of these cases were found to have partial necrosis of the bronchial mucosa. All of our patients underwent right-sided surgery that included subcarinal LND, as previously reported $(3,18)$.

LND, including both superior and inferior mediastinal lymph nodes, is widely performed as an adjunct to pulmonary resection for non-small cell lung cancer (2). This approach enables complete removal of all mediastinal lymph nodes and the surrounding fatty tissue (2). However, the importance of peribronchial tissue in wound healing is well known, and extensive LND may compromise bronchial microvascularization and negatively influence the healing of the bronchial stump (2). Impaired vascularization of the airway as a result of dissection and mobilization, systemic vascular disease, or (chemo)radiation as induction therapy are risk factors for impaired healing of an anastomosis, resulting in breakdown of the anastomotic and formation of a fistula $(6,19)$. Despite meticulous dissection of peritracheal or peribronchial tissue, appropriate preoperative nutritional and physiotherapy programs, and vascularized tissue covering, healing of the anastomosis and bronchial stump can be disturbed. All 7 patients in our study underwent subcarinal LND, and the bronchial stump or anastomosis was reinforced using the intercostal muscle flap in 4 patients, the pedicled thymus flap in 1 patient, and the parietal pleural flap in 1 patient. These reinforcement strategies were used for the purpose of preventing extension of empyema into the bronchial fistula or allowing a blood supply to the adjacent peribronchial tissue and bronchus as 
Table 3 Comparison of reports in the literature

\begin{tabular}{|c|c|c|c|c|c|c|c|c|c|c|}
\hline Year & Study & Number & Objective & Diagnosis & $\begin{array}{l}\text { Onset (POD) } \\
\text { [range] }\end{array}$ & $\begin{array}{l}\text { Pressure } \\
\text { (atm) }\end{array}$ & $\begin{array}{l}\text { Time } \\
\text { (min) }\end{array}$ & $\begin{array}{l}\text { Duration, } \\
\text { days [range] }\end{array}$ & $\begin{array}{l}\text { Recovery } \\
{[\%]}\end{array}$ & Adverse event \\
\hline 2011 & $\begin{array}{l}\text { Benhamed } \\
\text { et al. }\end{array}$ & 34 & Lob + ND2a2 & $\begin{array}{l}\text { BF at } 7,12 \\
\text { POD }\end{array}$ & $8 \pm 3$ & 2.5 & 90 & $14[1-48]$ & 28 [82] & 2 (barotrauma) \\
\hline 2015 & $\begin{array}{l}\text { Dickhoff } \\
\text { et al. }\end{array}$ & 1 & Carinoplasty & $\begin{array}{l}\text { BF at } 15 \\
\text { POD }\end{array}$ & 15 & 2.5 & 80 & 40 & $1[100]$ & 0 \\
\hline
\end{tabular}

BF, fiberoptic bronchoscope; Lob, lobectomy; N, number of cases; ND, nodal dissection; POD, postoperative days.

well as the healing wound.

HBOT consists of breathing $100 \%$ oxygen at a pressure higher than ambient pressure $(101.3 \mathrm{kPa})$ while the patient is inside a sealed treatment chamber. With such doses of oxygen, numerous beneficial, cellular, and physiologic effects can be achieved (20). HBOT is used primarily in the treatment of diving-related conditions, such as decompression sickness and cerebral arterial gas embolism. HBOT is also known to be an effective therapy for carbon monoxide poisoning and anaerobic infection. More recently, HBOT has been shown to have a supplementary therapeutic effect in the treatment of other disorders characterized by local ischemia (21), but has been a little evaluated specifically in POIB. The beneficial effects of hyperbaric oxygen occur via several mechanisms, including hyperoxygenation, proliferation of fibroblasts, deposition of collagen, angiogenesis, and vasculogenesis. Animal studies of tracheal transplantation have demonstrated several of these mechanisms, including an increase in the number, size, and distance of growth of blood vessels after HBOT $(10,11,22,23)$. HBOT has also been used for the clinical treatment of anastomotic complications related to tracheal resection and reconstruction, where it may help in healing and avoid tracheostomy $(13,24,25)$, and as an adjunctive treatment for POIB in patients with non-small cell lung cancer, where its effects seem to be promising $(12,14)$.

Once POIB and bronchial necrosis have developed, there is virtually no therapy that can support the recovery process, and repeat surgery to dismantle the anastomosis with loss of pulmonary tissue is often the only option. Although the evidence is based on animal studies reporting on HBOT after tracheal anastomosis $(11,26)$ and on its use for compromised flaps and grafts in plastic surgery (9), we decided to offer our patients this treatment. Six of our 7 cases had coverage of the bronchial stump and anastomosis with intercostal muscle, thymus or pleural tissue. Whether the eventual healing of the anastomosis was the result of HBOT or from ingrowth of the muscle flap and subsequent neovascularization, or both, could not be clarified in this study. To date, the rationale for this treatment is not completely understood, but several mechanisms have been proposed to explain how it promotes neovascularization and epithelialization and improves healing (8). Treatment should start before the "point of no return," that is, when ischemia has not yet progressed into full-thickness, and thus irreversible necrosis.

There are two reports on the value of HBOT for POIB after lung cancer surgery (Table 3). Benhamed et al. reported on the incidence of POIB after resection of primary lung cancer and the effect of HBOT on the outcome in 2011 (12). They reported that POIB occurred at a mean of $8 \pm 3$ days following surgery, and resolved in 28 patients $(82.3 \%)$ after application of HBOT. In their study, POIB worsened in 6 patients $(17.6 \%)$ and required surgical intervention, consisting of thoracostomy in 4 patients, lobectomy in 1 patient, and lobectomy and thoracostomy in 1 patient. Dickhoff $e t$ al. also published a case report of a patient who underwent extended pulmonary resection with carinoplasty for a centrally localized lung cancer with subsequent partial necrosis of one of the anastomoses that was successfully treated by HBOT (14). They used HBOT to facilitate normal wound healing because the patient's clinical condition remained excellent. Their patient showed the first signs of improvement after 27 days of HBOT, which was continued for 40 days with bronchoscopic surveillance. Candas et al. reported that HBOT may be effective in tracheal reconstruction by increasing epithelization and neovascularization after extended tracheal resection (23). Ariza-Prota et al. also described a patient with complicated tracheal radionecrosis who developed a tracheal ulceration with a small left posterior wall fissure extending into the mediastinum that was successfully treated with HBOT, local 
debridement, and antibiotics (25). Higuchi et al. investigated the safety and efficacy of HBOT for septic complications of lung transplantation (27). The 9 patients in their study received HBOT at a median of 56 days (range, $1-4,245$ days) following lung transplantation; 5 had complete resolution with excellent long-term graft function and two ceased therapy because of ear barotrauma $(n=1)$ and seizure without any permanent damage $(n=1)$. The positive effects on angiogenesis and local ischemia-reperfusion injury may be of benefit in the early post-lung transplantation period (27). In our present series, the median duration of the HBOT sessions was 7 days. After HBOT, POIB resolved in 5 (71\%) of 7 patients. Two patients experienced worsening POIB that required surgery. One patient developed tympanic barotrauma without any functional consequences. The most severe ischemic area was observed not at the anastomosis but at the bronchial wall on the distal side at the orifice of the right $\mathrm{B} 8, \mathrm{~B} 9$ and $\mathrm{B} 10$ on POD 158 in case 6. This finding underscores the need to consider the duration of HBOT for POIB.

\section{Study limitations}

One is its retrospective single-center design, which means that our findings may not necessarily be generalizable to more complex clinical settings and that prospective studies are needed to determine the merits of HBOT for POIB. There was also a degree of selection bias in that the study only included patients with confirmed symptomatic POIB and not patients with asymptomatic POIB. Therefore, the effect of HBOT might be evaluated more difficult to improve ischemia of asymptomatic POIB. Another limitation of our study was that we could not demonstrate the benefit of HBOT, so a prospective study with a control group is now needed.

\section{Conclusions}

HBOT can be considered for ischemic bronchitis found at the bronchial anastomosis or stump during postoperative bronchoscopic surveillance. In cases of reversible ischemia or partial necrosis of the bronchial stump and anastomosis, HBOT may prevent further progression to transmural ischemia, breakdown of the anastomosis, and development of a fistula at the stump. Currently, there are few human data supporting the use of HBOT for this specific indication. However, because of the few side effects and scarcity of alternative options, we recommend commencing this treatment urgently when there is doubt about the viability of the bronchus when monitored by frequent bronchoscopy. HBOT may be a promising therapeutic option for ischemic bronchitis, although further clinical trials are necessary to demonstrate the true benefits of HBOT for POIB.

\section{Acknowledgements}

None.

\section{Footnote}

Conflicts of Interest: The authors have no conflicts of interest to declare.

Ethical Statement: The study was approved by the institutional review board, Yamagata University, Japan (S-31) and written informed consent was obtained from all patients.

\section{References}

1. Couraud L, Nashef SA, Nicolini P, et al. Classification of airway anastomotic healing. Eur J Cardiothorac Surg 1992;6:496-7.

2. Watanabe S, Asamura H. Lymph node dissection for lung cancer: significance, strategy, and technique. J Thorac Oncol 2009;4:652-7.

3. Asamura H, Naruke T, Tsuchiya R, et al. Bronchopleural fistulas associated with lung cancer operations. Univariate and multivariate analysis of risk factors, management, and outcome. J Thorac Cardiovasc Surg 1992;104:1456-64.

4. Sirbu H, Busch T, Aleksic I, et al. Bronchopleural fistula in the surgery of non-small cell lung cancer: incidence, risk factors, and management. Ann Thorac Cardiovasc Surg 2001;7:330-6.

5. Sonobe $M$, Nakagawa $M$, Ichinose $M$, et al. Analysis of risk factors in bronchopleural fistula after pulmonary resection for primary lung cancer. Eur J Cardiothorac Surg 2000;18:519-23.

6. Regnard JF, Perrotin C, Giovannetti R, et al. Resection for tumors with carinal involvement: technical aspects, results, and prognostic factors. Ann Thorac Surg 2005;80:1841-6.

7. Yamamoto R, Tada H, Kishi A, et al. Effects of preoperative chemotherapy and radiation therapy on human bronchial blood flow. J Thorac Cardiovasc Surg 2000;119:939-45. 
8. Sander AL, Henrich D, Muth CM, et al. In vivo effect of hyperbaric oxygen on woundangiogenesis and epithelialization. Wound Repair Regen 2009;17:179-84.

9. Thom SR. Hyperbaric oxygen: its mechanisms and efficacy. Plast Reconstr Surg 2011;127 Suppl 1:131S-141S.

10. Gorur R, Hahoglu A, Uzun G, et al. Effects of hyperbaric oxygen therapy on wound healing after tracheal resection and end-to-end anastomoses in rats: results of early observations. Thorac Cardiovasc Surg 2008;56:359-62.

11. Celik B, Meydan AD, Kefeli M, et al. The effects of hyperbaric oxygen treatment on the healing of tracheal anastomosis following irradiation in rats. Thorac Cardiovasc Surg 2010;58:481-5.

12. Benhamed L, Bellier J, Fournier C, et al. Postoperative ischemic bronchitis after lymph node dissection and primary lung cancer resection. Ann Thorac Surg 2011;91:355-9.

13. Stock C, Gukasyan N, Muniappan A, et al. Hyperbaric oxygen therapy for the treatment of anastomotic complications after tracheal resection and reconstruction. J Thorac Cardiovasc Surg 2014;147:1030-5.

14. Dickhoff C, Daniels JM, van den Brink A, et al. Does hyperbaric oxygen therapy prevent airway anastomosis from breakdown? Ann Thorac Surg 2015;99:682-5.

15. Amin MB, Edge AB, Greene FL, et al. American Joint Committee on Cancer. AJCC Cancer Staging Manual. 8th ed. New York, NY: Springer, 2016.

16. Mathieu D. Contraindications to hyperbaric oxygen therapy. In: Neuman TS, Thom SR, eds. Physiology and Medicine of Hyperbaric Oxygen Therapy. 1st ed. Amsterdam, The Netherlands: Elsevier, 2008:587-98.

17. Satoh Y, Okumura S, Nakagawa K, et al. Postoperative ischemic change in bronchial stumps after primary lung cancer resection. Eur J Cardiothorac Surg 2006;30:172-6.
18. Cerfolio RJ. The incidence, etiology, and prevention of postresectional bronchopleural fistula. Semin Thorac Cardiovasc Surg 2001;13:3-7.

19. Mitchell JD, Mathisen DJ, Wright CD, et al. Resection for bronchogenic carcinoma involving the carina: long-term results and effect of nodal status on outcome. J Thorac Cardiovasc Surg 2001;121:465-71.

20. Tibbles PM, Edelsberg JS. Hyperbaric-oxygen therapy. N Engl J Med 1996;334:1642-8.

21. Hampson NB, Holm JR, Wreford-Brown CE, et al. Prospective assessment of outcomes in 411 patients treated with hyperbaric oxygen for chronic radiation tissue injury. Cancer 2012;118:3860-8.

22. Gould LJ, May T. The science of hyperbaric oxygen for flaps and grafts. Surg Technol Int 2016;28:65-72.

23. Candas F, Gorur R, Haholu A, et al. Is tracheal transplantation possible with cryopreserved tracheal allograft and hyperbaric oxygen therapy? An experimental study. Ann Thorac Surg 2016;101:1139-44.

24. De Wolf J, Fournier C, Surmei E, et al. Conservatively treated extended tracheal necrosis complicating pharyngolaryngectomy. Ann Thorac Surg 2015;99:1810-1.

25. Ariza-Prota M, Morales A, Grajeda J, et al. Successful healing of tracheal radionecrosis: role of hyperbaric oxygen therapy. Chest 2016;150:e147-50.

26. Azevedo LA, Parra RS, Da Rocha JJ, et al. Hyperbaric oxygen on the healing of ischemic colonic anastomosisan experimental study in rats. Undersea Hyperb Med 2010;37:405-11.

27. Higuchi T, Oto T, Millar IL, et al. Preliminary report of the safety and efficacy of hyperbaric oxygen therapy for specific complications of lung transplantation. J Heart Lung Transplant 2006;25:1302-9.
Cite this article as: Endoh M, Oizumi H, Kato H, Suzuki J, Watarai H, Hamada A, Suzuki K, Nakahashi K, Shiono S, Sadahiro M. Hyperbaric oxygen therapy for postoperative ischemic bronchitis after resection of lung cancer. J Thorac Dis 2018;10(11):6176-6183. doi: 10.21037/jtd.2018.10.45 